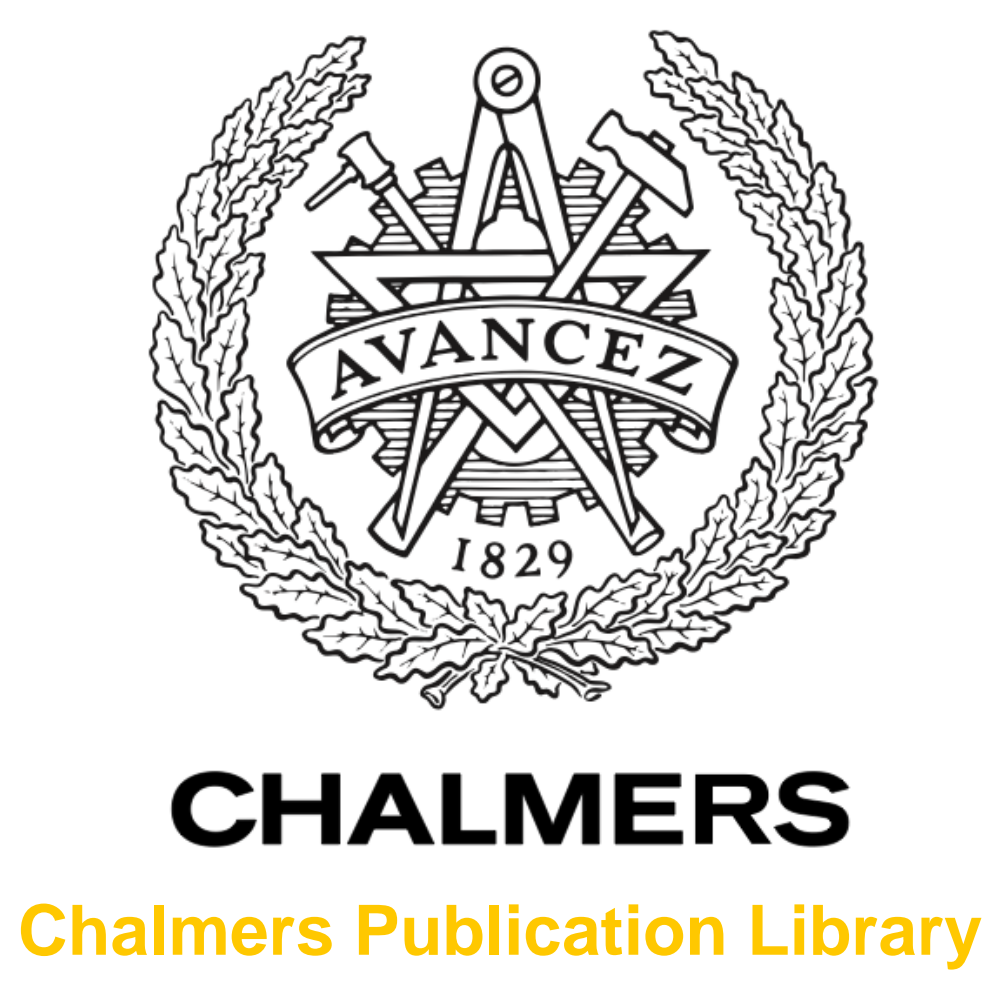

\title{
Dynamic equations for fluid-loaded porous plates using approximate boundary conditions
}

This document has been downloaded from Chalmers Publication Library (CPL). It is the author's version of a work that was accepted for publication in:

Journal of the Acoustical Society of America (ISSN: 0001-4966)

Citation for the published paper:

Folkow, P. ; Johansson, M. (2009) "Dynamic equations for fluid-loaded porous plates using approximate boundary conditions". Journal of the Acoustical Society of America, vol.

125(5), pp. 2954-66.

http://dx.doi.org/10.1121/1.3086267

Downloaded from: http://publications.lib.chalmers.se/publication/104186

Notice: Changes introduced as a result of publishing processes such as copy-editing and formatting may not be reflected in this document. For a definitive version of this work, please refer to the published source. Please note that access to the published version might require a subscription.

Chalmers Publication Library (CPL) offers the possibility of retrieving research publications produced at Chalmers University of Technology. It covers all types of publications: articles, dissertations, licentiate theses, masters theses, conference papers, reports etc. Since 2006 it is the official tool for Chalmers official publication statistics. To ensure that Chalmers research results are disseminated as widely as possible, an Open Access Policy has been adopted.

The CPL service is administrated and maintained by Chalmers Library. 
Dynamic equations for a fluid-loaded porous plate using approximate boundary conditions

\author{
Peter D. Folkow ${ }^{\mathrm{a})}$ and Martin Johansson \\ Department of Applied Mechanics, \\ Chalmers University of Technology, \\ SE-412 96 Göteborg, \\ Sweden.
}

(Dated: June 16, 2008)

Fluid-loaded porous plate 1 


\begin{abstract}
Approximate boundary equations for fluid-loaded thin poroelastic layers are derived for time harmonic conditions. The layer is modeled according to Biot theory and both open and closed pores conditions at the fluid-porous interfaces are considered. Series expansions in the thickness variable are used to replace the porous field variables at the boundaries by their values at the center-plane of the layer. When truncated, this yields a system of equations with the fluid pressures at the surfaces and the porous field variables at the center-plane as unknowns. The problem is split into separate symmetric and antisymmetric cases and is solved by utilizing the governing equations for the porous medium. The result is two $2 \mathrm{D}$ differential equations in the plane of the layer which relates the fluid pressures to their normal derivatives at the surfaces. Numerical comparisons are made with 3D Biot theory for two material configurations and two thicknesses. The agreement was found to be better than expected. A thin porous layer may then in some cases be replaced by the approximate boundary conditions and thereby simplify the analysis of fluid-porous coupled problems.
\end{abstract}

PACS numbers: 


\section{INTRODUCTION}

In this paper approximate boundary conditions for fluid-loaded saturated porous plates are derived. Their intended use is as replacement for the porous layer in acoustic-poroelastic coupled problems.

Porous layers are found in several disciplines; biomechanics, seismology, geomechanics and acoustics. In reality the structure of porous media is very complex and irregular. For macroscopic scales, i.e. when the relevant length scales are much larger than the pore sizes, a widely used theory was formulated by Biot ${ }^{1}$. It is a linear theory that treats the poroelastic material as a continuum with averaged macroscopic displacement fields and is formulated with a set of effective parameters. Much work has been devoted to the determination of these parameters since the theory was first presented. This theory is, however, still fairly complicated and in many situations simplifications may be justified.

The complexity of porous materials has motivated much work on approximations and simplified methods. One approach is to use a simplified material model such as the rigid frame model ${ }^{2,3}$. In many situations the geometries of the porous structures are such that there is room for other simplifying approximations. Several authors such as Taber ${ }^{4}$, Theodorakopoulos and $\operatorname{Beskos}^{5}$, and Leclaire et al. ${ }^{6}$, have derived approximate plate equations for porous plates. They rely on the Kirchhoff hypothesis combined with the Biot theory and in some cases assume predominant transverse fluid flow. Numerical solutions to these equations for clamped porous plates were obtained, applying Galerkin's variational method, by Leclaire et al. ${ }^{7}$ for acoustical excitation and Etchessahar et al. ${ }^{8}$ for a concentrated force. The case of in-plane fluid flow only was treated by Li.et al. ${ }^{9}$. Another possibility is to develop semi-empirical equations, e.g. Lee et al. ${ }^{10}$ and Bliss ${ }^{11}$.

This paper focuses on deriving approximate boundary conditions for fluid-loaded thin porous plates. The main object is to replace the porous layer by two differential equations in the surface plane, expressed in terms of the exterior fluid variables. These differential

a)Electronic address: peter.folkow@chalmers.se 
equations constitute the approximate boundary conditions and account for the effect of the porous layer. It is thus not necessary to model the porous medium, which may greatly simplify the analysis of porous-fluid coupled problems. The approach is based on series expansions in the thickness coordinate and the result is a two-dimensional representation of the porous layer, much like plate theories. The present method applies for the derivation of porous plate equations as well. This will be the subject of a future paper.

Effective boundary conditions to first order in the thickness was studied by Bövik ${ }^{12,13}$ for various configurations of fluids and elastic layers. The method was generalized by Johansson et $a l{ }^{?}$ where approximate boundary conditions for a fluid-loaded elastic plate were given up to order five in the thickness. This method is closely related to those presented by Boström et $a l{ }^{?}$ and $\operatorname{Losin}^{14,15}$ who used series expansions to derive plate equations for isotropic elastic plates. An other example is the work of Johansson and Niklasson? who treated piezoelectric anisotropic elastic media. In the present paper the results of Johansson et al. are generalized to poroelastic media.

When deriving the equations it is assumed that linear theory is valid and that the layer thickness is, at most, of the order of a typical wavelength. As the porous layer is modeled using Biot theory, it is also assumed that the characteristic pore size is much smaller than the thickness so that the porous medium may be considered as a continuum. No further assumptions regarding the behavior of the porous variables are made. Since the approximate boundary conditions are given in terms of Biot parameters it is possible to adapt the approximate boundary conditions to the problem at hand and, if justified, simplify them further.

The paper is organized as follows: The relevant equations of the Biot theory are first presented in Sec. II. In Sec. ?? an outline of the derivation method is given, followed by the derivation of the approximate boundary equations for the cases of closed and open pores. Numerical results are presented in Sec. IV, where the reflection coefficient is calculated for an incident plane wave at various angles of incidence by means of the approximate boundary equations. The results are compared to the full three dimensional Biot theory. The paper 
is sumarized in Sec. V.

\section{ASSUMPTIONS AND GOVERNING EQUATIONS}

Consider an infinite, isotropic porous plate of thickness $h$ immersed in a fluid, see Fig. (1). The saturated plate is modelled according to the Biot theory ${ }^{1}$, where the densitities of the solid and fluid parts are $\rho_{s}$ and $\rho_{f}$, respectively. The state of the plate may be expressed through the solid macroscopic displacement $\mathbf{u}$ together with the average displacement $\mathbf{U}$ and the pressure $p$ of the liquid phase. The state of the surrounding fluid, density $\rho_{0}$, is modelled through the pressure $p_{0}$ and the displacement $\mathbf{U}^{0}$. Viscous effects are neglected in the exterior fluid, but they are important in the porous domain.

For a thin layer approximation, the plate thickness is assumed to be smaller than the shortest wavelength considered. However, for the continuum theory to hold, the plate thickness is large when compared to the pore size. These assumptions are in line with the lowfrequency theory presented in ${ }^{1}$ where the flow in the pores are considered to obey Poiseuille flow.

The pores at the plate boundaries are either closed or fully open; the intermediary states are not considered here. With closed pores it is understood that a thin, impermeable massless layer separates the exterior fluid from the fluid in the plate. The properties of the saturating fluid and the surrounding fluid may in such a case differ. In the case of open pores, fluid may flow between the plate and the surroundings through the boundaries. It is then understood that the saturating fluid is identical to the surrounding fluid.

Biot theory supports three bulk wave types. One shear wave (wave speed $c_{s}$ ) and two compressional waves referred to as the fast and slow compressional waves (wave speeds $c_{p 1}$ and $c_{p 2}$ respectively). Here, it is the slowest wave that is the critical one for thin layer approximations. Which of the three waves that is the slowest depends on the frame and saturating fluid materials as well as the frequency, but in most cases $c_{p 2}<c_{s}<c_{p 1}$. 


\section{A. Governing Equations}

Considering time harmonic conditions, the equations of motion governing the displacements in porous media may be written as ${ }^{1}$

$$
\begin{array}{r}
N \nabla^{2} \mathbf{u}+\nabla \nabla \cdot((N+A) \mathbf{u}+Q \mathbf{U})=-\omega^{2}\left(\rho_{11} \mathbf{u}+\rho_{12} \mathbf{U}\right) \\
\nabla \nabla \cdot(Q \mathbf{u}+R \mathbf{U})=-\omega^{2}\left(\rho_{12} \mathbf{u}+\rho_{22} \mathbf{U}\right)
\end{array}
$$

where the factor $\mathrm{e}^{-\mathrm{i} \omega t}$ has been omitted. Here $N$ is the shear modulus of the frame and $Q, A$, and $R$ are generalized elastic coefficients. These latter factors are related to the measurable quantities the porosity $\Phi$, the bulk modulus of the frame $K_{b}$, the bulk modulus of the solid material $K_{s}$, and the bulk modulus of the fluid $K_{f}$, see Allard ${ }^{2}$ :

$$
\begin{aligned}
& A=\frac{(1-\Phi)\left(1-\Phi-K_{b} / K_{s}\right) K_{s}+\Phi K_{s} K_{b} / K_{f}}{C_{K}}-\frac{2}{3} N, \\
& Q=\frac{\Phi\left(1-\Phi-K_{b} / K_{s}\right) K_{s}}{C_{K}}, \quad R=\frac{\Phi^{2} K_{s}}{C_{K}} .
\end{aligned}
$$

where

$$
C_{K}=1-\Phi-K_{b} / K_{s}+\Phi K_{s} / K_{f}
$$

The densities $\rho_{i j}$, are defined by

$$
\rho_{12}=(1-\alpha) \Phi \rho_{f}, \quad \rho_{11}=(1-\Phi) \rho_{s}-\rho_{12}, \quad \rho_{22}=\Phi \rho_{f}-\rho_{12},
$$

where the density $\rho_{12}$ represents added mass due to coupling of the solid and fluid motions. Here $\alpha$ is the dynamic tortuosity, related to both the porosity and the geometry of the interconnected pores. For viscous flow in the pores causing damping, $\alpha$ is complex and frequency dependent according to the model by Johnson et al. ${ }^{16}$

$$
\alpha=\alpha_{\infty}+\frac{\mathrm{i} \eta \Phi}{\rho_{f} \kappa_{0} \omega}\left(1-\frac{4 \mathrm{i} \alpha_{\infty}^{2} \kappa_{0}^{2} \rho_{f} \omega}{\eta \Phi^{2} \Lambda^{2}}\right)^{1 / 2}
$$

where $\eta$ is the viscosity, $\kappa_{0}$ is the static permeability and $\Lambda$ is a characteristic length of the pore size. For non-viscous fluids the tortuosity is given by the constant value $\alpha=\alpha_{\infty}$. This

choice of notation is due to the limit $\omega \rightarrow \infty$ for $\alpha$ in the viscous case. Due to the beavior of $\alpha$ for viscous flow the densities $\rho_{i j}$ are hereby also complex and frequency dependent. Note 
that when the saturating fluid is a gas, thermal effects should be considered as the thermal conduction modifies the bulk modulus of the fluid. Such effects are included in the model through the complex and frequency dependent $K_{f}$ by $^{2}$

$$
K_{f}=\frac{\gamma_{h} p_{\mathrm{atm}}}{\gamma_{h}-\left(\gamma_{h}-1\right)\left(1+\mathrm{i} \frac{\omega_{0}}{\omega}\left(1-\frac{\mathrm{i} \omega}{2 \omega_{0}}\right)^{1 / 2}\right)^{-1}}, \quad \omega_{0}=\frac{\sigma}{\rho_{f} \alpha_{\infty} c^{2} \operatorname{Pr}}
$$

Here, $\operatorname{Pr}$ denotes the Prantl-number, $p_{\text {atm }}$ is the atmospheric pressure, $\gamma_{h}$ is the ratio of specific heats, $\sigma$ is the flow resistivity and $c$ is a form factor which depends on the shape of the pores.

The constitutive equations for the stresses in the porous plate are

$$
\begin{aligned}
& \boldsymbol{\sigma}^{s}=N\left(\nabla \mathbf{u}+(\nabla \mathbf{u})^{T}\right)+\mathbf{I} \nabla \cdot(A \mathbf{u}+Q \mathbf{U}), \\
& \boldsymbol{\sigma}^{f}=(-\mathbf{I} \Phi p)=\mathbf{I} \nabla \cdot(Q \mathbf{u}+R \mathbf{U}),
\end{aligned}
$$

where $\mathbf{I}$ is the $3 \times 3$ identity tensor. The stresses are the averaged stress of the solid frame $\boldsymbol{\sigma}^{s}$ and the averaged stress of the fluid portion $\boldsymbol{\sigma}^{f}$. The sum of these stresses forms the total macroscopic stress $\boldsymbol{\sigma}=\boldsymbol{\sigma}^{s}+\boldsymbol{\sigma}^{f}$.

In the exterior fluid the governing equation for the pressure is according to the simple wave equation

$$
\left(\nabla^{2}+k_{0}^{2}\right) p_{0}=0, \quad k_{0}=\omega / c_{0}
$$

where $c_{0}$ is the wave velocity and $k_{0}$ is the wave number for waves in the fluid. The relation between the pressure $p_{0}$ and the displacement $\mathbf{U}^{0}$ in the surrounding fluid is given by the momentum equation

$$
\nabla p_{0}=\rho_{0} \omega^{2} \mathbf{U}^{0}
$$

\section{B. Boundary Conditions}

The boundary conditions at $z= \pm h / 2$ for a porous-fluid interface stem from continuity of traction, continuity of filtration velocity as well as Darcy's law ${ }^{17}$. The continuity requirements for the surface tractions in tangential and normal directions are obtained using Eqs. 
(7) and (8)

$$
\begin{gathered}
N\left(\partial_{x} u_{z}+\partial_{z} u_{x}\right)=0 \\
N\left(\partial_{y} u_{z}+\partial_{z} u_{y}\right)=0 \\
2 N \partial_{z} u_{z}+\nabla \cdot((A+Q) \mathbf{u}+(Q+R) \mathbf{U})=-p_{0} .
\end{gathered}
$$

Here, the partial derivatives are expressed $\partial_{x}=\partial / \partial_{x}$ and so on. The fluid flow across the interfaces $z= \pm h / 2$ are governed by continuity of the normal component of the filtration vector and by Darcy's law. Under time harmonic conditions these relations are

$$
\begin{gathered}
\Phi\left(U_{z}-u_{z}\right)=U_{z}^{0}-u_{z}, \\
\pm \mathrm{i} \omega \Phi\left(U_{z}-u_{z}\right)=\kappa_{s}\left(p_{0}-p\right),
\end{gathered}
$$

where the parameter $\kappa_{s}$ characterizes the permeability of the interface.

The state of the porous plate at the boundaries may be given in terms of either the fields $\{\mathbf{u}, \mathbf{U}\}$ or $\{\mathbf{u}, p\}$, while for the surrounding fluid the boundary conditions are given by either $\mathbf{U}^{0}$ or $p_{0}$. The choice is a matter of convenience and here the $\left\{\mathbf{u}, \mathbf{U}, p_{0}\right\}$-formulation is used in conformity with the governing equations for the porous plate Eq. (1) and the surrounding fluid Eq. (9). Thus, the pressure of the liquid phase $p$ appearing in Darcy's law Eq. (15) may be rewritten using Eq. (8). In a similar manner, the surrounding fluid displacement $U_{z}^{0}$ in Eq. (14) may be written in terms of the pressure field $p_{0}$ adopting Eq. (10). Since the results of the fluid flow boundary conditions Eqs. (14) and (15) are fundamentally different for closed and open pores, these cases are discussed separately below.

For closed pores the permeability $\kappa_{s}$ is zero as no mass transport is allowed between the exterior fluid and the fluid of the porous medium. Darcy's law Eq. (15) then reduces to $U_{z}=u_{z}$ at the surfaces. This simplifies the filtration condition Eq. (14) to $U_{z}^{0}=u_{z}$ as expected. By using Eq. (10), the interface fluid flow relations become

$$
\begin{aligned}
\partial_{z} p_{0} & =\rho_{0} \omega^{2} u_{z}, \\
0 & =U_{z}-u_{z} .
\end{aligned}
$$


In the case of open pores the permeability $\kappa_{s}$ is infinite. Due to finiteness of the flow, Darcy's law Eq. (15) gives $p=p_{0}$ at the boundaries. Adopting Eqs. (10) and (8) gives the interface fluid flow relations

$$
\begin{gathered}
\partial_{z} p_{0}=\rho_{0} \omega^{2}\left((1-\Phi) u_{z}+\Phi U_{z}\right) \\
p_{0}=-\Phi^{-1} \nabla \cdot(Q \mathbf{u}+R \mathbf{U})
\end{gathered}
$$

Here, $\rho_{f}=\rho_{0}$ as the same fluid is assumed inside and outside the plate.

\section{PLATE EQUATIONS}

\section{A. Series expansions}

There are different ways to obtain plate theories; either based on different kinematical assumptions in line with the classical theories for elastic plates, or in a more rigorous fashion adopting the three dimensional equations of motion. Here, a systematic approach will be used based on the three dimensional equations of motion together with power series expansions of the physical fields with respect to the thickness coordinate $z$. This method is believed to be asymptotically correct without any ad hoc assumptions, resulting in a hierarchy of higher order plate theories that can (in principle) be truncated to any order. One such expansion method is to expand the plate displacement fields around the midplane $z=0$, which has been done for an isotropic elastic plate, see Boström et al. ${ }^{18}$. Another method is to expand the boundary conditions around the midplane $z=0$. This approach

has been adopted by Johansson et al. ${ }^{19}$ for a fluid-loaded elastic plate, where the results are expressed in terms of so called approximate boundary conditions. These two methods seem to be analogous, which is discussed in the case of an elastic plate ${ }^{19}$.

In the present case for fluid-loaded porous plates, the latter approach is used in order to benefit from the results from fluid-loaded elastic plate. Hence, the plate equations are expressed in terms of approximate boundary conditions, where the plate fields are eliminated so as to obtain differential equations in terms of the fluid pressure $p_{0}$ and its normal deriva- 
tive $\partial_{z} p_{0}$ at the boundaries. By doing this, the influence from the porous plate is present implicitly in the differential equations, without the need of solving the plate equations explicitly in term of the plate fields. For clarity, the plate equations will also be expressed So, when using series expansion of the boundary conditions in order to eliminate the plate fields, it is convenient to proceed in terms of the differences and sums of the boundary fields

$$
\Delta f=f(x, y, h / 2)-f(x, y,-h / 2), \quad \Sigma f=f(x, y, h / 2)+f(x, y,-h / 2),
$$

where $f=\left\{\mathbf{u}, \mathbf{U}, p_{0}\right\}$ and their spatial derivatives. For the plate fields $\mathbf{u}$ and $\mathbf{U}$ and their spatial derivatives, the sums and differences are expanded in Maclaurin series in the thickness coordinate $z$

$$
\begin{aligned}
& \Delta f=2 \sum_{j=0}^{n-1} \frac{\partial_{z}^{2 j+1} f_{c}}{(2 j+1) !}\left(\frac{h}{2}\right)^{2 j+1}+\mathcal{O}\left(h^{2 n+1}\right), \\
& \Sigma f=2 \sum_{j k=0}^{n-1} \frac{\partial_{z}^{2 j} f_{c}}{(2 j) !}\left(\frac{h}{2}\right)^{2 j}+\mathcal{O}\left(h^{2 n}\right),
\end{aligned}
$$

where $\partial_{z}^{m} f_{c}=\left.\partial_{z}^{m} f(x, y, z)\right|_{z=0}$. The sums and differences of the boundary conditions for the tractions Eqs. (11)-(13) and the fluid flow Eqs. (16)-(17) (closed pores) or Eqs. (18)-(19) (open pores) may then be written in terms of the sums and differences of the exterior fluid pressure $p_{0}$ and $\partial_{z} p_{0}$, together with the porous field variables at the center plane $\mathbf{u}_{c}$ and $\mathbf{U}_{c}$.

In conformity with Johansson et $a .^{19}$, two separate cases are identified due to the differential orders in the normal direction of the plate displacements. For $\Delta p_{0}$ and $\Sigma \partial_{z} p_{0}$, even order $z$-derivatives $\partial_{z}^{2 m}$ act on the fields $\left\{u_{z, c}, U_{z, c}\right\}$ and their derivatives in the $(x y)$ plane, while odd order $z$-derivatives $\partial_{z}^{2 m+1}$ act on the fields $\left\{u_{x, c}, u_{y, c}, U_{x, c}, U_{y, c}\right\}$ and their derivatives in the $(x y)$-plane. Considering $\Sigma p_{0}$ and $\Delta \partial_{z} p_{0}$ the opposite situation holds. The explicit representation of the plate fields $\left\{\mathbf{u}_{c}, \mathbf{U}_{c}\right\}$ and their spatial derivatives may then be eliminated in order to give separate differential equations in terms of $\Delta p_{0}$ and $\Sigma \partial_{z} p_{0}$ (antisymmetric case) and $\Sigma p_{0}$ and $\Delta \partial_{z} p_{0}$ (symmetric case). For each such case, $8 m+7$ equations must be solved if the expansions in the plate thickness $h$ are to involve terms up to and including $h^{2 m+1}$. Since there are only five boundary conditions, the remaining equations are 
found through the governing equations Eq. (1). By performing normal derivatives of these equations evaluated at the center plane, $8 m+2$ additional equations are obtained.

The solutions of the equation systems discussed above resulting in the approximate boundary conditions, may be written in terms of differential operators. For closed pores these boundary conditions are expressed

$$
\begin{aligned}
& \left(\sum_{j=0}^{n-1} h^{2 j} \omega^{2} \mathcal{C}_{1, j}\right) \Delta p_{0}=\left(\sum_{j=1}^{n} h^{2 j-1} \mathcal{C}_{2, j}\right) \Sigma \partial_{z} p_{0}+\mathcal{O}\left(h^{2 n}\right) \\
& \left(\sum_{j=2}^{n} h^{2 j} \omega^{2} \mathcal{C}_{3, j}\right) \Sigma p_{0}=\left(\sum_{j=2}^{n} h^{2 j-1} \mathcal{C}_{4, j}\right) \Delta \partial_{z} p_{0}+\mathcal{O}\left(h^{2 n+1}\right),
\end{aligned}
$$

for the antisymmetric and symmetric cases, respectively while for open pores the equations are

$$
\begin{aligned}
& \left(\sum_{j=1}^{n-1} h^{2 j} \mathcal{C}_{5, j}\right) \Delta p_{0}=\left(\sum_{j=1}^{n-1} h^{2 j+1} \mathcal{C}_{6, j}\right) \Sigma \partial_{z} p_{0}+\mathcal{O}\left(h^{2 n}\right) \\
& \left(\sum_{j=2}^{n} h^{2 j} \mathcal{C}_{7, j}\right) \Sigma p_{0}=\left(\sum_{j=1}^{n-1} h^{2 j+1} \mathcal{C}_{8, j}\right) \Delta \partial_{z} p_{0}+\mathcal{O}\left(h^{2 n+1}\right)
\end{aligned}
$$

in the antisymmetric and symmetric cases, respectively. The differential operators $\mathcal{C}_{i, j}$ generally involve $\nabla_{s}^{2 p} \omega^{2 j-2 p}$ for $p=0, \ldots, j$ with $\nabla_{s}^{2}=\partial_{x}^{2}+\partial_{y}^{2}$. Closed form representations of $\mathcal{C}_{i, j}$ involve a substantial number of different terms even at low order. It should be noted that for a series expansion involving terms up to and including order $h^{2 m+1}$ in Eqs. (21) and (22), even higher order terms appear in the elimination process. However, as these higher order terms are not stable when using even more terms in the series expansion, the equations are truncated. Thus, only terms up to and including order $h^{2 m+1}$ are present in the final result given above. In the present work, series expressions involving terms up to and including order $h^{5}$ are used in the numerical results. These lengthy closed form expressions, obtained through tedious calculations using the mathematical software MATHEMATICA, are not presented here. The explicit representations given below involve terms up to and including order $h^{3}$. 


\section{B. Closed pores}

Expressing the relations (23) and (24) explicitly for terms up to and including $h^{3}$, the approximate boundary condition for the antisymmetric case becomes

$$
\begin{aligned}
& \beta_{1} k_{t}^{2}\left\{1-\frac{1}{2}\left(\frac{h}{2}\right)^{2}\left[3 \nabla_{s}^{2}+3 k_{\mathrm{av}}^{2}\right]\right\} \Delta p_{0} \\
& =\left\{\frac{h}{2} k_{t}^{2}-\frac{1}{6}\left(\frac{h}{2}\right)^{3}\left[8\left(1-\gamma_{1}\right) \nabla_{s}^{4}+\left(16 k_{s}^{2}+7 k_{t}^{2}-24 k_{\mathrm{av}}^{2}-8 k_{1}^{2}+8 k_{p 1}^{2} k_{p 2}^{2} / k_{s}^{2}\right) \nabla_{s}^{2}\right.\right. \\
& \left.\left.+\left(2 k_{s}^{2}+3 k_{\mathrm{av}}^{2}\right) k_{t}^{2}+2 \gamma_{2} k_{p 1}^{2} k_{p 2}^{2}\right]\right\} \Sigma \partial_{z} p_{0},
\end{aligned}
$$

while the approximate boundary condition for the symmetric case is

$$
\begin{aligned}
& \beta_{1} k_{t}^{2}\left(\nabla_{s}^{2}+k_{p 1}^{2}\right)\left(\nabla_{s}^{2}+k_{p 2}^{2}\right)\left\{\frac{h}{2}-\frac{1}{6}\left(\frac{h}{2}\right)^{3}\left[3 \nabla_{s}^{2}+3 k_{\mathrm{av}}^{2}\right]\right\} \Sigma p_{0} \\
& =\left\{\left[-4\left(1-\gamma_{1}\right) \nabla_{s}^{4}-\left(k_{t}^{2}-4 k_{1}^{2}\right) \nabla_{s}^{2}-\gamma_{2} k_{p 1}^{2} k_{p 2}^{2}\right]\right. \\
& +\frac{1}{6}\left(\frac{h}{2}\right)^{2}\left[12\left(1-\gamma_{1}\right) \nabla_{s}^{6}+\left(5 k_{t}^{2}+12\left(1-\gamma_{1}\right) k_{\mathrm{av}}^{2}-12 k_{1}^{2}\right) \nabla_{s}^{4}\right. \\
& \left.\left.+\left(\left(9 k_{\mathrm{av}}^{2}-2 k_{s}^{2}\right) k_{t}^{2}-12 k_{1}^{2} k_{\mathrm{av}}^{2}+3 \gamma_{2} k_{p 1}^{2} k_{p 2}^{2}\right) \nabla_{s}^{2}+\left(2 k_{t}^{2}+3 \gamma_{2} k_{\mathrm{av}}^{2}\right) k_{p 1}^{2} k_{p 2}^{2}\right]\right\} \Delta \partial_{z} p_{0} .
\end{aligned}
$$

Here $k_{s}, k_{p 1}$, and $k_{p 2}$ are the bulk wave numbers of the shear wave, the fast compressional wave, and the slow compressional wave, respectively. They are given by

$$
k_{p 1, p 2}^{2}=\frac{\omega^{2}}{c_{p 1, p 2}^{2}}=\frac{2 a_{1} \omega^{2}}{a_{2} \mp \sqrt{a_{2}^{2}-4 a_{1} a_{3}}}, \quad k_{s}^{2}=\frac{\omega^{2}}{c_{s}^{2}}=\frac{a_{1} \omega^{2}}{N \rho_{22}},
$$

where the constants $a_{i}$ are

$$
a_{1}=\rho_{11} \rho_{22}-\rho_{12}^{2}, \quad a_{2}=R \rho_{11}-2 Q \rho_{12}+S \rho_{22}, \quad a_{3}=R S-Q^{2},
$$

using $S=2 N+A$. The wave number $k_{t}$ is a generalized shear wave number, $k_{\text {av }}$ is the mean square of the bulk wave numbers, and $k_{1}$ is an auxiliary wave number. They are defined by

$$
k_{t}^{2}=\frac{\rho_{t} \omega^{2}}{N}, \quad k_{\mathrm{av}}^{2}=\frac{k_{p 1}^{2}+k_{p 2}^{2}+k_{s}^{2}}{3}, \quad k_{1}^{2}=\frac{\left((Q+R) \rho_{12}-(Q+S-N) \rho_{22}\right) \omega^{2}}{a_{3}} .
$$

The density $\rho_{t}$ is the total density of the saturated porous body, according to

$$
\rho_{t}=\rho_{11}+2 \rho_{12}+\rho_{22}=(1-\Phi) \rho_{s}+\Phi \rho_{f}
$$


All the squared wave numbers in Eqs. (27) and (28) have a positive real part. The symbols $\beta_{1}, \gamma_{1}$ and $\gamma_{2}$ are non-dimensional numbers (with positive real part for the porous materials studied numerically in this paper) expressed in terms of densities and generalized elastic coefficients according to

$$
\beta_{1}=\frac{\rho_{0}}{\rho_{t}}, \quad \gamma_{1}=\frac{N R}{a_{3}}, \quad \gamma_{2}=\frac{R+2 Q+S}{N}
$$

Comparing Eqs. (27) and (28) with the corresponding approximate boundary equations for an elastic layer reported by Johansson et al. ${ }^{19}$, it is seen that the expressions are quite similar. The differences are most pronounced for the symmetric case, where the derivatives in the porous case are two orders higher than in the elastic case. This has to do with the fact that Biot's theory supports three bulk waves, and evidently all of these are important to order $h^{3}$ in Eq. (28). In conformity with Johansson et al. ${ }^{19}$, it is possible to factorize the compressional bulk wave operator in the symmetric case. As there are two such waves in the porous case, the factorized operator is $\left(\nabla_{s}^{2}+k_{p 1}^{2}\right)\left(\nabla_{s}^{2}+k_{p 2}^{2}\right)$ on the left hand side of Eq. (28). This implies that when the surface component of the wave number of the fluid loading coincides with the wave numbers of any of the compressional bulk waves in the porous medium, no symmetric normal motion of the fluid-porous boundary is generated. In the porous layer a guided pressure wave may propagate with the wave speed of any of the compressional bulk wave speeds as if the porous domain occupied the full three dimensional space. Moreover, the symmetrical part of the fluid loading behaves as if the fluid-porous interfaces were rigid walls. It should be noted, though, that the compressional bulk wave numbers are complex when losses are included in the porous medium. This coincidence phenomenon thus does not occur since viscosity is neglected in the exterior fluid.

It is not difficult to generalize Eqs. (27) and (28) to the case of different exterior fluids as this only influences the normal displacement continuity condition, Eq. (16). The right hand side of this equation may still be expressed in terms of either the sum or the difference of the normal displacement of the porous solid. Denoting the density of the exterior fluids on the positive and negative $z$ by $\rho_{0}$ and $\rho_{0}^{\prime}$, respectively, this is done by substituting the 
right hand sides of Eqs. (28) and (27) according to

$$
\begin{aligned}
\Sigma \partial_{z} p_{0} & \rightarrow \frac{1}{2}\left(\left(1+\rho_{0} / \rho_{0}^{\prime}\right) \Sigma \partial_{z} p_{0}+\left(1-\rho_{0} / \rho_{0}^{\prime}\right) \Delta \partial_{z} p_{0}\right), \\
\Delta \partial_{z} p_{0} & \rightarrow \frac{1}{2}\left(\left(1-\rho_{0} / \rho_{0}^{\prime}\right) \Sigma \partial_{z} p_{0}+\left(1+\rho_{0} / \rho_{0}^{\prime}\right) \Delta \partial_{z} p_{0}\right) .
\end{aligned}
$$

It is instructive to see whether the results for a homogeneous plate derived by Johansson et $a l .{ }^{19}$ are retrieved when the porosity tends to zero, $\Phi \rightarrow 0$. Starting with the generalized elastic coefficients Eq. (2) and the densities Eq. (4), these become $N=\mu, A=\lambda, \rho_{11}=\rho_{s}$ while $Q=R=0$ and $\rho_{12}=\rho_{22}=0$ in the limit. Here, $\mu$ and $\lambda$ are the Lamé constants for a homogeneous plate. The bulk wave numbers in (29) are hereby $k_{p 1}^{2}=\omega^{2} / c_{p}^{2}, k_{s}^{2}=\omega^{2} / c_{s}^{2}$ and $k_{p 2}^{2}=0$, where now $c_{p}^{2}=\mu / \rho_{s}$ and $c_{s}^{2}=(\lambda+2 \mu) / \rho_{s}$. Moreover, the auxiliary wave number in (31) becomes $k_{1}=0$ while the constants in (33) become $\gamma_{1}=1 / \gamma_{2}=\gamma$ where $\gamma=\mu /(\lambda+2 \mu)$. Comparing these equations to the ones given in Johansson et al. ${ }^{19}$, a few terms differ. However, by closer inspection of the resulting non-truncated porous equations using a $h^{3}$ series expansion, some operators may actually be exactly factorized when the porosity tends to zero. Such factorized operators stem from the procedure of reducing the number of porous plate parameters to the homogeneous case. Hence, by eliminating these common operators, identical results are obtained when the porosity tends to zero as for the homogeneous plate ${ }^{19}$. The factorized operators in question are

$$
\left(1-\frac{1}{2}\left(\frac{h}{2}\right)^{2} \nabla_{s}^{2}\right), \quad \nabla_{s}^{2}\left(1-\frac{1}{6}\left(\frac{h}{2}\right)^{2} \nabla_{s}^{2}\right),
$$

in the antisymmetric and symmetric case, respectively.

$$
\begin{aligned}
& \beta_{1} k_{t}^{2}\left\{\nabla_{s}^{2}+k_{2}^{2}\right\} \Delta p_{0} \\
& =\left\{\frac{h}{2}\left[k_{s}^{2}\left(\nabla_{s}^{2}+k_{2}^{2}\right)\right]-\frac{1}{6}\left(\frac{h}{2}\right)^{3}\left[8\left(1-\gamma_{1}\right) \nabla_{s}^{6}\right.\right. \\
& \left.\left.+\left(8\left(1-2 \gamma_{1}\right)\left(k_{s}^{2}\left(1+\gamma_{1}\right)-3 k_{\mathrm{av}}^{2}\right)-8 k_{p 1}^{2} k_{p 2}^{2} / k_{s}^{2}\right) \nabla_{s}^{4}\right]\right\} \Sigma \partial_{z} p_{0},
\end{aligned}
$$

where wave numbers and constants are defined by Eqs. (29)-(33). This equation has the same structure as for the case of an elastic layer. It is noted that to order $h$ this is the 
momentum equation for an incompressible layer of density $\rho_{t}$, with zero shear stiffness, as it may be written as $\Delta p_{f}=h \rho_{t} \omega^{2} \Sigma u_{z} / 2$, i.e. only the inertial effect is included.

\section{Open pores}

Expressing the relations (25) and (26) explicitly for terms up to and including $h^{3}$, the approximate boundary condition for the antisymmetric case becomes

$$
\begin{aligned}
& \beta_{2}\left\{k_{2}^{2}-\frac{1}{6}\left(\frac{h}{2}\right)^{2}\left[8\left(1-\gamma_{1}\right) \Phi^{2} \nabla_{s}^{4}+\left(16 \Phi^{2} k_{s}^{2}+7 k_{2}^{2}-24 \Phi^{2} k_{\mathrm{av}}^{2}-8 k_{3}^{2}+8 \Phi^{2} k_{p 1}^{2} k_{p 2}^{2} / k_{s}^{2}\right) \nabla_{s}^{2}\right.\right. \\
& \left.\left.+\left(2 k_{s}^{2}+3 k_{\mathrm{av}}^{2}\right) k_{2}^{2}+2 \gamma_{3} k_{p 1}^{2} k_{p 2}^{2}\right]\right\} \Delta p_{0} \\
& =\left\{\frac{h}{2} k_{s}^{2}-\frac{1}{6}\left(\frac{h}{2}\right)^{3}\left[8\left(1-\gamma_{1}\right) \nabla_{s}^{4}+\left(13-8 \gamma_{1}\right) k_{s}^{2} \nabla_{s}^{2}+\left(2 k_{s}^{2}+3 k_{\mathrm{av}}^{2}\right) k_{s}^{2}\right]\right\} \Sigma \partial_{z} p_{0},
\end{aligned}
$$

while the approximate boundary condition for the symmetric case is

$$
\begin{aligned}
& \beta_{2}\left\{\left(\frac{h}{2}\right)\left[4\left(1-\gamma_{1}\right) \Phi^{2} \nabla_{s}^{4}+\left(k_{2}^{2}-4 k_{3}^{2}\right) \nabla_{s}^{2}+\gamma_{3} k_{p 1}^{2} k_{p 2}^{2}\right]\right. \\
& -\frac{1}{6}\left(\frac{h}{2}\right)^{3}\left[12\left(1-\gamma_{1}\right) \Phi^{2} \nabla_{s}^{6}+\left(5 k_{2}^{2}+12\left(1-\gamma_{1}\right) \Phi^{2} k_{\mathrm{av}}^{2}-12 k_{3}^{2}\right) \nabla_{s}^{4}\right. \\
& \left.\left.+\left(\left(9 k_{\mathrm{av}}^{2}-2 k_{s}^{2}\right) k_{2}^{2}-12 k_{3}^{2} k_{\mathrm{av}}^{2}+3 \gamma_{3} k_{p 1}^{2} k_{p 2}^{2}\right) \nabla_{s}^{2}+\left(2 k_{2}^{2}+3 \gamma_{3} k_{\mathrm{av}}^{2}\right) k_{p 1}^{2} k_{p 2}^{2}\right]\right\} \Sigma p_{0} \\
& =\left\{\left[-4\left(1-\gamma_{1}\right) \nabla_{s}^{4}-k_{s}^{2}\right]\right. \\
& +\frac{1}{6}\left(\frac{h}{2}\right)^{2}\left[20\left(1-\gamma_{1}\right) \nabla_{s}^{4}+\left(12\left(3-\gamma_{1}\right) k_{\mathrm{av}}^{2}-\left(1+8 \gamma_{1}\right) k_{s}^{2}-8 k_{p 1}^{2} k_{p 2}^{2} / k_{s}^{2}\right) \nabla_{s}^{4}\right. \\
& \left.\left.\left.+\left(9 k_{\mathrm{av}}^{2}-2 k_{s}^{2}\right) k_{s}^{2}\right)\right]\right\} \Delta \partial_{z} p_{0} .
\end{aligned}
$$

Some new auxiliary wave numbers and non-dimensional numbers enter here, in addition to those defined by Eqs. (29)-(33),

$$
\begin{aligned}
& \beta_{2}=\frac{\rho_{0}}{\rho_{22}}, \quad \gamma_{3}=\frac{R-2 \Phi(Q+R)}{N}+\Phi^{2} \gamma_{2}, \\
& k_{2}^{2}=\frac{\left(\rho_{22}-2 \Phi^{2} \rho_{f}\right) \omega^{2}}{N}+\Phi^{2} k_{t}^{2}, \quad k_{3}^{2}=\Phi k_{4}^{2}+\Phi^{2} k_{1}^{2}, \quad k_{4}^{2}=\frac{\left(Q \rho_{22}-R \rho_{12}\right) \omega^{2}}{a_{3}} .
\end{aligned}
$$

As in the closed pore case these terms have a positive real part. By inspection of Eqs. (36)(37) it is seen that the same type of differential operators appear as in the closed pore case 
Eqs. (27)-(28), albeit in a somewhat more complicated way. Moreover, some of the lefthand side operators in the open pore case happen to be generalizations of the corresponding operators appearing on the right-hand side in the closed pore case. Contrary to Eq. (28), it is not possible to make factorization of the left-hand side of the symmetric equation Eq. (37), due to the filtration effects at the surfaces of the porous layer.

$$
\begin{aligned}
& \beta_{1} k_{t}^{2}\left(1-\Phi^{2} \beta_{3}\right)\left\{\left(k_{t}^{2}+2 \Phi \beta_{4} k_{4}^{2}\right) \nabla_{s}^{2}+k_{t}^{2} k_{2}^{2}\right\} \Delta p_{0} \\
& =\left\{\frac{h}{2}\left[\left(\left(1-\Phi^{2} \beta_{2}\right) k_{s}^{2}+2 \Phi \beta_{2}\left(1-\Phi^{2} \beta_{3}\right) k_{4}^{2}\right) \nabla_{s}^{2}+\left(1-\Phi^{2} \beta_{2}\right) k_{s}^{2} k_{2}^{2}\right]\right. \\
& \left.-\frac{1}{6}\left(\frac{h}{2}\right)^{3}\left(1-\Phi^{2} \beta_{2}\right)\left[8\left(1-\gamma_{1}\right) \nabla_{s}^{6}+\left(8\left(1-2 \gamma_{1}\right) k_{2}^{2}-8 k_{p 1}^{2} k_{p 2}^{2} / k_{s}^{2}\right) \nabla_{s}^{4}\right]\right\} \Sigma \partial_{z} p_{0},
\end{aligned}
$$

where $\beta_{4}=\rho_{t} / \rho_{22}$.

\section{NUMERICAL RESULTS}

The asymptotic approximate boundary conditions are to be compared to the threedimensional Biot theory as well as to the plate theory according to Theodorakopoulos and Beskos $^{5}$, see Eq .... Two material combinations are considered: water and QF-20, a quartzfiber studied by Johnson et $a l .{ }^{20}$, and air in combination with a plastic foam studied by Allard et al. ${ }^{2,21}$. The material data of importance for these combinations are given in Table I. For both material combinations the saturating and the exterior fluids are hereafter assumed to be the same, but viscous effects are accounted for only in the saturating fluid. In the foam-air case also thermal effects are considered for the saturating fluid, as well as damping in the plastic foam.

Due to the viscous effects resulting in frequency dependent material parameters, all three bulk waves are dispersive; the higher the frequency, the higher the velocity. Dispersion is particularly important for the slow compressional wave, whereas the other two modes are virtually unaffected. This is manifested in Fig. 2 where curves are presented for the three

waves when the real part of the wavelengths equal $2 h$. For the plastic foam case, the slow 
compressional wave velocity actually becomes higher than the shear wave for higher frequencies. The choice of wavelength used in Fig. 2 corresponds to the first positive interference maximum for normal incidence, which could be seen as a reference upper frequency limit for the shortest wavelength according to the approximate theories. Besides being dispersive, the considered porous media are also dissipative. The damping is most pronounced for the slow compressional wave in the low-frequency region; the other waves show dissipation that generally increase with frequency. Due to these frequency dependent effects, it is thus not possible to introduce a nondimensional frequency variable so that the results are independent of the thickness. Therefore two different thicknesses, $h=0.4 \mathrm{~m}$ and $h=0.04 \mathrm{~m}$ corresponding to different frequency intervals, have been chosen in the numerical results ${ }^{22}$. Hereby both the low frequency dissipative regime and the high frequency propagating regime of the slow compressional wave are investigated. These thicknesses are also indicated in Fig. 2 as solid lines.

To validate the approximate plate equations presented above, the transmission and absorption coefficients are calculated. Consider $z<-h / 2$ and let plane waves propagate in the $(x z)$-plane towards $z=-h / 2$, giving rise to reflected and transmitted plane waves. Thus, the incident, reflected and transmitted waves may be expressed as

$$
\begin{array}{ll}
p_{0, i}=\mathrm{e}^{\mathrm{i}\left(k_{0}\left(\zeta x+\sqrt{1-\zeta^{2}} z\right)-\omega t\right)} & z<-h / 2, \\
p_{0, r}=R \mathrm{e}^{\mathrm{i}\left(k_{0}\left(\zeta x-\sqrt{1-\zeta^{2}} z\right)-\omega t\right)} & z<-h / 2, \\
p_{0, t}=T \mathrm{e}^{\mathrm{i}\left(k_{0}\left(\zeta x+\sqrt{1-\zeta^{2}} z\right)-\omega t\right)} & z>h / 2,
\end{array}
$$

where $\zeta=\sin \phi ; \phi$ being the angle measured from the normal to the plate boundaries.

It is now straightforward to solve for $R$ and $T$ using the exact three-dimensional equations of motion Eq. (1) with pertinent boundary conditions. For the asymptotic equations, either Eqs. (27)-(28) or Eqs. (36)-(37) are to be used. As the loading is such that purely antisymmetric or symmetric modes will not be generated in the general case, the antisymmetric and symmetric equations are solved as a system. The numerical results involve solutions based on the asymptotic $h$ and $h^{3}$ expansions, respectively. The range of appli- 
cability for each truncation level is hereby clearly visible. As for the T-B theory Eq. ???, these equations are solved postulating only antisymmetric motion, resulting in a simple relation between $R$ and $T$. The reflection and transmission coefficients are used to derive the absorption coefficient $A_{d}=1-|R|^{2}-|T|^{2}$.

It is convenient to introduce the nondimensional frequency $\Omega=k_{s, \infty} h$ where $k_{s}=\omega / c_{s, \infty}$. Here $c_{s, \infty}$ is the limit $\omega \rightarrow \infty$ for $c_{s}$ which corresponds to the case of a non-viscous fluid. Note that the real part of $c_{s, \infty}$ is considered in the plastic foam case, as the complex shear modulus $N$ results in a complex velocity.

\section{A. Quartz-fiber and water}

In the case of QF-20 and water $c_{s, \infty} \approx 2036 \mathrm{~m} \mathrm{~s}^{-1}$. From Fig. 2(a) it is seen that for the slow compressional wave the limit $k_{p 2} h=\pi$ occurs for $h=0.4 \mathrm{~m}$ when $\omega \approx 2250 \mathrm{rad} \mathrm{s}^{-1}$ which corresponds to $\Omega \approx 0.44$, and for $h=0.04 \mathrm{~m}$ when $\omega \approx 70000 \mathrm{rad} \mathrm{s}^{-1}$ corresponding to $\Omega \approx 1.38$. For the shear wave $k_{s} h=\pi$ occurs when $\Omega \approx 2.96$ for both thicknesses.

\section{Closed pores}

The results for closed pores are presented in Figure 3. The first two Figs. 3(a) and 3(b) show the modulus of $T$ when the angle of incidence is $\phi=45^{\circ}$. The results using the $h^{3}$ expansion are more accurate than the asymptotic $h$ theory as expected. It is more surprising that the $h$ theory is superior to the T-B theory which involves terms of order $h^{3}$. This probably stems from the importance of the symmetric modes not modelled in the $\mathrm{T}-\mathrm{B}$ flexural plate theory. Considering the curves for the $h^{3}$ expansion, they deviate from the exact curves around $\Omega \approx 1$ for $h=0.04 \mathrm{~m}$ and around $\Omega \approx 1.5$ for $h=0.4 \mathrm{~m}$, respectively. This latter case is far beyond the upper limit for the slow compressional wave discussed above. This accuracy may be connected to the fact that in this low frequency interval the slow wave is much attenuated. It should also be stated that the slow compressional wave generally is not much excited when the pores are $\operatorname{closed}^{23}$. For other angles of incidence, 
similar results as depicted in Figs. 3(a) and 3(b) are obtained.

The dependence of $|T|$ upon the angle of incidence is presented in Fig. 3(c) for the frequency $\Omega=0.8$ when $h=0.04 \mathrm{~m}$. The accuracy of the different approximations show a similar behavior as in the previous figures. Note the rapid transition of the transmission coefficient around $\zeta \approx 0.45$, that is $\phi \approx 27^{\circ}$. This is associated with the first compressional mode closely related to the zeroth symmetric Lamb mode; a similar behavior is reported by Johansson et al. ${ }^{19}$ for a fluid-loaded elastic plate. The phenomenon is not captured by the $\mathrm{T}-\mathrm{B}$ theory since it does not take symmetric motion into account. Another minor incorrectness with the $\mathrm{T}-\mathrm{B}$ equation is that $|T|=1$ for the grazing angle, $\zeta=1$. The exact and asymptotic equations predict that the transmission modulus falls quickly to zero as $\zeta$ approaches unity. Similar plots are obtained for $h=0.4 \mathrm{~m}$ and for other frequencies, albeit the lower frequencies $\Omega<0.5$ result in pronounced transmission for most angles as expected. Note that the angle of incidence corresponding to the rapid transition of the transmission coefficient varies little with frequency as this symmetric wave guide mode exhibits small dispersion.

In Fig. 3(d) the absorption coefficient is plotted against the angle of incidence when $\Omega=0.8$ and $h=0.04 \mathrm{~m}$. Here the influence of the zeroth symmetrical mode is clearly visible, showing its significant influence on layer absorption. As for the transmission coefficient the $\mathrm{T}-\mathrm{B}$ theory does not identify this behavior. Note here that the $h$-expansion is actually slightly superior to the asymptotic $h^{3}$ theory; a situation that also appear for other frequencies. Similar plots are obtained for other frequencies as well as for $h=0.4 \mathrm{~m}$ where in the latter case the absorption coefficient is slightly more pronounced.

\section{Open pores}

The results for open pores are presented in Figure 4. Here the first two Figs. 4(a) and 4(b) show the modulus of $T$ when the angle of incidence is $\phi=15^{\circ}$ and $\phi=45^{\circ}$, respectively, for $h=0.04 \mathrm{~m}$. As for closed pores, the $h^{3}$ expansion is more accurate than the asymptotic 
$h$ and the T-B theories. All approximate theories deviate from the exact curves at lower frequencies when compared to the closed pore case. The main reason for this is probably due to that the slow compressional wave is more excited when the pores are open ${ }^{23}$. According to the $h^{3}$ expansion for $\phi=15^{\circ}$ no transmission occurs at $\Omega \approx 1.5$, whereas the exact solution shows almost zero transmission at $\Omega \approx 2.2$. Such behavior are also present for $\phi=45^{\circ}$. A similar situation appeared for heavy loading on an elastic plate ${ }^{19}$. However, this does not imply total reflection in the porous case as absorption is present. For thicker plates, less dramatic curves are obtained. Here the transmission coefficient is generally larger, and the T-B theory is more accurate; sometimes even better than the asymptotic $h^{3}$ theory.

The dependence of $|T|$ upon the angle of incidence is presented in Fig. 4(c) for the frequency $\Omega=0.8$ when $h=0.04 \mathrm{~m}$. The accuracy of the different approximations and the rapid transition show a similar behavior as for closed pores, clearly resembling the results for heavy loading on an elastic plate ${ }^{19}$. It is noted that the transition jump is more pronounced while there is less variation with the angle of incidence in the open pore case.

In Fig. 4(d) the absorption coefficient is plotted against the angle of incidence when $\Omega=0.8$ and $h=0.04 \mathrm{~m}$. Here the $h^{3}$ expansion theory is visibly superior to the $h$ theory. When compared to the closed pore case, the absorption is more pronounced here, which probably is due to that the slow compressional wave is much less excited in the former case. Similar plots are obtained for $h=0.4 \mathrm{~m}$ as well as for other frequencies where in the latter case the absorption coefficient is larger for higher frequencies.

Note from the results in Figs. 3 and 4 that the T-B solutions scarcely depend on the boundary conditions; virtually the same results are obtained for closed and open pores, seen fro Eq. ????

\section{B. Plastic foam and air}

Consider next the material configuration where the frame is made of a plastic foam of high flow resistivity saturated with air. This combination is studied by Allard et al. ${ }^{21}$. In 
addition to viscous effects, viscoelastic and thermal effects are included as well. In this case, the material parameters in Eq (2) may be simplified as $K_{f} / K_{s} \ll 1$ and $K_{b} / K_{s} \ll 1$. Hence, the parameters may be approximated $b^{2}$

$$
Q=K_{f}(1-\Phi), \quad A=K_{b}+\frac{(1-\Phi)^{2}}{\Phi} K_{f}-\frac{2}{3} N, \quad R=\Phi K_{f}
$$

where $K_{f}$ is according to (6). The bulk modulus of the frame is assumed to be modeled in line with an isotropic elastic material

$$
K_{b}=\frac{2 N(1+\nu)}{3(1-2 \nu)},
$$

where $\nu$ the Poisson's ratio. The dynamic tortousity (5) is obtained from the material constants in Table I using ${ }^{2}$

$$
\Lambda^{2}=\frac{8 \alpha_{\infty} \eta}{\Phi \sigma c^{2}}, \quad \kappa_{0}=\eta / \sigma
$$

For this material combination $c_{s, \infty} \approx 76 \mathrm{~m} \mathrm{~s}^{-1}$. From Fig. 2(b) it is seen that for the slow compressional wave the limit $k_{p 2} h=\pi$ occurs for $h=0.4 \mathrm{~m}$ when $\omega \approx 70 \mathrm{rad} \mathrm{s}^{-1}$ which corresponds to $\Omega \approx 0.91$, and for $h=0.04 \mathrm{~m}$ when $\omega \approx 6000 \operatorname{rad~s}^{-1}$ corresponding to $\Omega \approx 3.13$. For the shear wave $k_{s} h=\pi$ occurs when $\Omega \approx 3.13$ for both thicknesses. By inspection, all three bulk wave speeds in the porous material are here lower than the speed of sound in the surrounding air. Moreover, the shear wave is now slower than the slow compressional wave for high frequencies.

\section{Closed pores}

The results for closed pores are presented in Figure 5. Fig. 5(a) shows the modulus of $T$ when the angle of incidence is $\phi=45^{\circ}$ and $h=0.04$. All approximate theories render good results; the $\mathrm{T}-\mathrm{B}$ theory being superior in the interval $\Omega \approx 2$ to $\Omega \approx 3$, after which the asymptotic theories are more accurate. For low frequencies, the $h^{3}$ expansion theory is the most accurate. Almost identical curves are obtained for the thicker layer $h=0.4$, implying that the influence of the slow compressional wave is limited, see discussion in the quartz-fiber case IV.A.1. For other angles of incidence, similar results are obtained. 
The dependence of $|T|$ upon the angle of incidence is presented in Fig. 5(b) for the frequency $\Omega=3$ when $h=0.04 \mathrm{~m}$. The accuracy of the different approximations show a similar behavior as in the previous figures. Contrary to the quartz-fiber case there is no rapid transition of the transmission coefficient. The reason for this is that such a coincidence

phenomenon with the zeroth symmetrical mode does not correspond to a real-valued angle of incidence; $\zeta \approx 2.45$. Similar plots are obtained for $h=0.4 \mathrm{~m}$ and for other frequencies, albeit the lower frequencies $\Omega<0.2$ result in pronounced transmission for most angles as expected. When comparing the transmission coefficient presented in Fig. 5(b) to the quartz-fiber case $3(\mathrm{c})$, it is clear that considerably less is transmitted in the foam-air case.

When studying the quite modest absorption coefficient in Fig. 5(c) when $h=0.04$ $\mathrm{m}$ and Fig. $5(\mathrm{~d})$ when $h=0.4 \mathrm{~m}$ for $\Omega=3$, it is clear that much of the wave is reflected considering the magnitude of $|T|$, see Fig. 5(b). Here, the T-B curves are hard to distinguish being placed almost on the horizontal coordinate line. Similar plots are obtained for other frequencies, where the magnitude of the absorption coefficient increases with frequency.

\section{Open pores}

The results for open pores are presented in Figure 6. Here the first Fig. 6(a) shows the modulus of $T$ when the angle of incidence is $\phi=45^{\circ}$ for $h=0.04 \mathrm{~m}$. Contrary to the closed pores case, the asymptotic expansion theories are more accurate than the $\mathrm{T}-\mathrm{B}$ theory in the interval considered. As for the material combination quartz-fiber and water, the approximate curves deviate from the exact curve at lower frequencies when compared to the closed pores case due to the more pronounced excitation of the slow compressional wave. Therefore a more narrow frequency interval is studied in 6(a) compared to 5(a). Similar curves are obtained for other angles and frequencies. For thicker plates the T-B theory is more accurate and sometimes even better than the asymptotic $h^{3}$ theory; compare similar situation for the case with QF-20 and water.

The dependence of $|T|$ upon the angle of incidence is presented in Fig. 6(b) for the 
frequency $\Omega=1.5$ when $h=0.04 \mathrm{~m}$. The accuracies of the different approximations show a similar behavior as in Fig. 6(a). It is noted that slightly more is transmitted when compared to the closed pores case Fig. 5(b).

The last two Figures 6(c) and 6(d) present the absorption coefficient against the angle of incidence when $\Omega=1.5$ for $h=0.04 \mathrm{~m}$ and $h=0.4 \mathrm{~m}$, respectively. When compared to the closed pore case, the absorption is more pronounced here mainly due to the influence of the slow compressional wave. As the absorption coefficient increases with frequency, the thin layer case depicted in Fig. 6(c) show less absorption than thick layer case in Fig. 6(d). Note that the T-B curves are almost on the horizontal coordinate line.

As for the quartz-fiber case, the T-B solutions scarcely depend on the boundary conditions (closed or open pores) for the porous foam and air case.

\section{CONCLUSIONS}

The approximate boundary conditions may be transformed into a space-time partial differential equations when frequency dependent viscous, viscoelastic, and thermal effects are neglible. In the general case the space-time domain approximate boundary conditions are partial integro-differential equations.

Approximate boundary conditions for a thin fluid-loaded porous layer are derived for open and closed pores under time-harmonic conditions. Series expansions in the thickness coordinate are utilized and boundary conditions up to order three in the thickness are presented. In both cases separate symmetric and antisymmetric equations are obtained when the surrounding fluids are the same. The closed pores case is generalized to different surrounding fluids, where the equations couple.

The equations have the same structure as the corresponding approximate boundary conditions for an elastic layer. However, sixth order tangential derivatives appear in the symmetric equations. A further similarity with the elastic case is that the differential operator for the symmetric pressure term for closed pores may be factorized so that the symmetric 
load term vanishes when the surface component of the wave number of the fluid loading coincides with the wave numbers of the compressional bulk waves.

Numerical results are compared with 3D Biot theory for two material configurations: quartz-fiber saturated with water and plastic foam saturated with air. The performances of the approximate boundary conditions are surprisingly good at wave-number-thickness products of order one. In many cases acceptable approximations are provided even by the much simpler $h^{1}$-theory. This is probably due to the damping of the slow compressional wave, which would otherwise limit the range of applicability as its wave speed is comparably low. Another explanation is that the influence from flexural plate motions here is less pronounced than for the case of an elastic layer, which in the latter case drastically limited the validity of the $h^{1}$-theory ${ }^{?}$. The agreement is better for closed pores. Again, this is believed to be due to the slow compressional wave, which is less excited when the pores are closed.

\section{ACKNOWLEDGMENT}

This work has been supported in part by the Swedish Research Council (VR) and in part by the Volvo-Chalmers Vehicle Research Program.

\section{APPENDIX: PARAMETERS OF POROUS MEDIA}

$$
\begin{gathered}
\frac{1}{2 N}\left\{1-\frac{1}{2}\left(\frac{h}{2}\right)^{2}\left[2\left(2-\gamma_{1}\right) \nabla_{s}^{2}+\left(k_{s}^{2}-k_{s}^{2}+k_{p 1}^{2} k_{p 2}^{2} / k_{s}^{2}\right)\right]\right\} \Delta p_{0} \\
\frac{1-\Phi \beta_{3}}{2 N}\left\{1-\frac{1}{6}\left(\frac{h}{2}\right)^{2}\left[\left(10\left(1-\gamma_{1}\right)+\gamma_{4}\right) \nabla_{s}^{2}+3 k_{\text {av }}^{2}+k_{s}^{2}\left(2+\gamma_{4}\right)\right]\right\} \Delta p_{0}
\end{gathered}
$$

where

$$
\gamma_{4}=\frac{N(\Phi Q-(1-\Phi) R)}{a_{3}\left(1-\Phi \beta_{3}\right)}
$$




\section{References}

1 M. A. Biot, "Theory of propagation of elastic waves in a fluid-saturated porous solid. I. Low-frequency range", J. Acoust. Soc Am. 28, 168-78 (1956).

2 J. F. Allard, Propagation of Sound in Porous Media (Elsevier Science Publishers Ltd, London) (1993).

3 P. M. Morse and K. U. Ingard, Theoretical Acoustics (McGraw-Hill, New York) (1968).

4 A. L. Taber, "A theory for transverse deflection of poroelastic plates", Trans. Am. Soc. Mech. Eng. 59, 628-634 (1992).

5 D. D. Theodorakopoulos and D. E. Beskos, "Flexural vibrations of poroelastic plates", Acta Mech. 103, 628-634 (1994).

6 P. Leclaire, K. V. Horoshenkov, and A. Cummings, "Transverse vibrations of a thin rectangular porous plate saturated by a fluid", J. Sound Vib. 247, 1-18 (2001).

7 P. Leclaire, K. V. Horoshenkov, M. J. Swift, and D. C. Hothersall, "The vibrational response of a clamped rectangular porous plate", J. Sound Vib. 247, 19-31 (2001).

8 M. Etchassahar, S. Sahraout, and B. Brouard, "Bending vibrations of a rectangular poroelastic plate", C. R. Acad. Sci. IIb 329, 615-620 (2001).

9 L. P. Li, G. Cederbaum, and K. Schulgasser, "Theory of poroelastic plates with in-plane diffusion", Int. J. Solids Struct. 34, 2515-4530 (1997).

10 J.-H. Lee and J. Kim, "Simplified method to solve sound transmission through structures lined with porous material", J. Acoust. Soc Am. 110, 2282-94 (2001), Pt. 1.

11 D. B. Bliss, "Study of the bulk reacting porous absorbers and a new boundary condition for thin porous layer", J. Acoust. Soc Am. 71, 533-45 (1982).

12 P. Bövik, "A comparison between the Tiersten model and $\mathrm{O}(h)$ boundary conditions for elastic surface waves guided by thin layers", Trans. Am. Soc. Mech. Eng. 63, 162-167 (1996).

13 P. Bövik and P. Olsson, "Effective boundary conditions for the scattering of twodimensional SH waves from a curved thin elastic layer", Proc. R. Soc. London, Ser. 
A 439, 257-269 (1992).

14 N. Losin, "Asymptotics of flexural waves in isotropic elastic plates", J. Appl. Mech. 64, 336-342 (1997).

15 N. Losin, "Asymptotics of extensional waves in isotropic elastic plates", J. Appl. Mech. 65, 1042-1047 (1998).

16 D. L. Johnson, J. Koplik, and R. Dashen, "Theory of dynamic permeability and tortuosity in fluid-saturated porous media", J. Fluid. Mech. 176, 379-402 (1987).

17 T. Bourbié, O. Coussy, and B. Zinsner, Acoustics of Porous Media (Technip., Paris) (1987).

18 A. Boström, G. Johansson, and P. Olsson, "On the rational derivation of a hierarchy of dynamic equations for a homogeneous, isotropic, elastic plate", Int. J. Solids Struct. 38, $2487-2501(2001)$.

19 M. Johansson, P. Folkow, A. Hägglund, and P. Olsson, "Approximate boundary conditions for a fluid-loaded elastic plate", J. Acoust. Soc. Am. 118, 3436-3446 (2005).

20 D. L. Johnson, T. J. Plona, and H. Kojima, "Probing porous media with first and second sound. II. Acoustic properties of water-saturated porous media", J. Appl. Phys. 76, 115125 (1994).

21 J.-F. Allard, C. Depolliér, and W. Lauriks, "Measurement and prediction of surface impedance at oblique incidence of a plastic foam of high flow resistivity", J. Sound Vib. 132, 51-60 (1989).

22 H. Belloncle, H. Franklin, F. Luppé, and J. Conoir, "Normal modes of a poroelastic plate and their relation to the reflection and transmission coefficients", Ultrasonics 41, 207-216 (2003).

23 P. N. J. Rasolofasaon, "Importance of interface hydraulic condition on the generation of second bulk compressional wave in porous media", Appl. Phys. Lett. 52, 780-782 (1988). 
TABLE I. Material data for QF-20 and water and plastic foam and air

Symbol Unit QF-20 and water Foam and air

\begin{tabular}{cccc}
\hline$\rho_{f}$ & $\mathrm{~kg} / \mathrm{m}^{3}$ & 1000 & 1.213 \\
$\rho_{s}$ & $\mathrm{~kg} / \mathrm{m}^{3}$ & 2759 & 429 \\
$\Phi$ & - & 0.402 & 0.93 \\
$\alpha_{\infty}$ & - & 1.89 & 3.2 \\
$N$ & $\mathrm{MPa}$ & 7.63 & $1.8(10-\mathrm{i})$ \\
$K_{f}$ & $\mathrm{MPa}$ & 2.22 & - \\
$K_{s}$ & $\mathrm{MPa}$ & 36.6 & - \\
$K_{b}$ & $\mathrm{MPa}$ & 9.47 & - \\
$\Lambda$ & $\mu \mathrm{m}$ & 19.0 & - \\
$\eta$ & $\mathrm{Pa} \mathrm{s}$ & $1.14 \times 10^{-3}$ & $1.84 \times 10^{-5}$ \\
$\kappa_{0}$ & $\mathrm{~m}^{2}$ & $1.68 \times 10^{-11}$ & - \\
$\sigma$ & $\mathrm{Nm}$ & & 0.71 \\
$c$ & - & - & $55 \times 10^{3}$ \\
$\nu$ & - & - & 3.37 \\
$\gamma_{h}$ & - & - & 0.4 \\
\hline \hline atm & $\mathrm{Mpa}$ & - & 0.10 \\
\hline
\end{tabular}




\section{List of Figures}

FIG. 1 Problem geometry . . . . . . . . . . . . . . . . 30

FIG. 2 Curves for $k h=\pi$, where $k$ is the real part of the wave numbers $k_{p 1}$ (solid), $k_{s}$ (dashed) and $k_{p 2}$ (dotted). The horizontal solid lines indicate the chosen

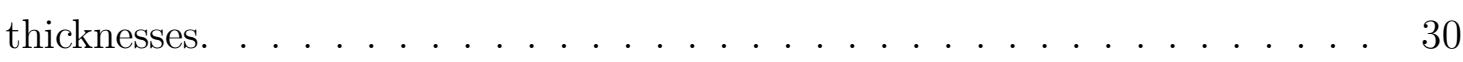

FIG. 3 Closed pores for QF-20 and water. — Exact, $---h^{3}, \cdots h,-\cdot-\mathrm{T}-\mathrm{B} . \quad 31$

FIG. 4 Open pores for QF-20 and water. — Exact, $---h^{3}, \cdots h,-\cdot-\mathrm{T}-\mathrm{B} . \quad 32$

FIG. 5 Closed pores for foam and air. _— Exact, $---h^{3}, \cdots h,-\cdot-$ T-B. . . 33

FIG. 6 Open pores for foam and air. Exact, $---h^{3}, \cdots h,-\cdot-$ T-B. . . 34 


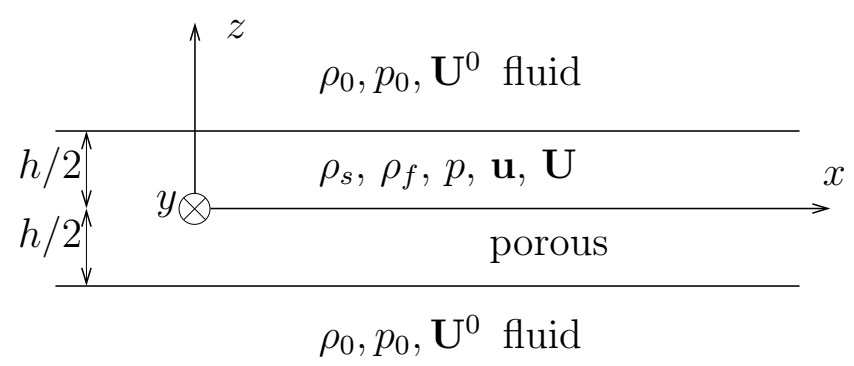

FIG. 1. Problem geometry

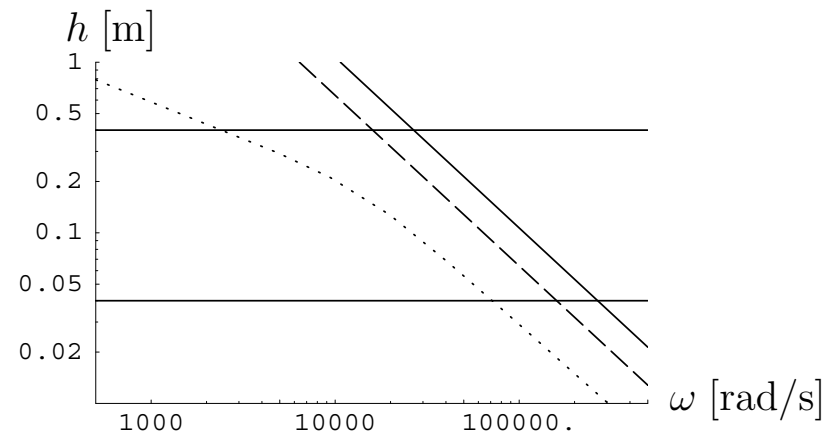

(a)Quartz-fiber and water

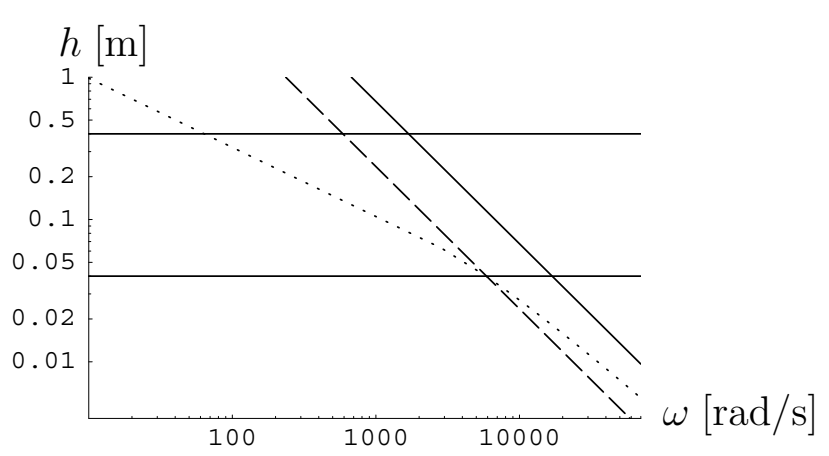

(b)Plastic foam and air

FIG. 2. Curves for $k h=\pi$, where $k$ is the real part of the wave numbers $k_{p 1}$ (solid), $k_{s}$ (dashed) and $k_{p 2}$ (dotted). The horizontal solid lines indicate the chosen thicknesses. 


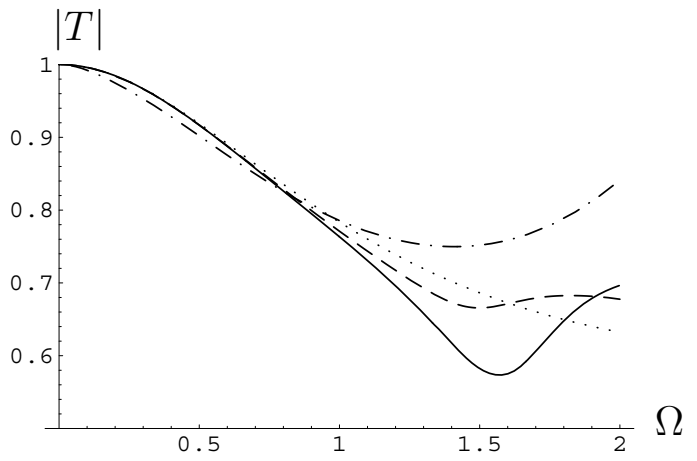

(a) $\phi=45^{\circ}, h=0.04 \mathrm{~m}$.

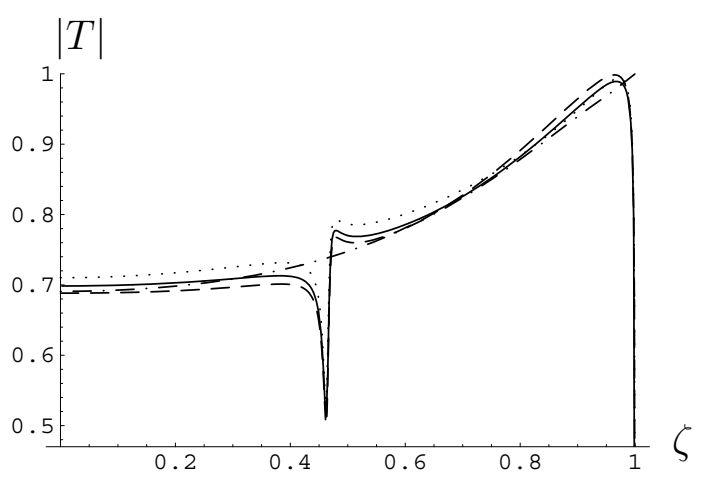

(c) $\Omega=0.8, h=0.04 \mathrm{~m}$.

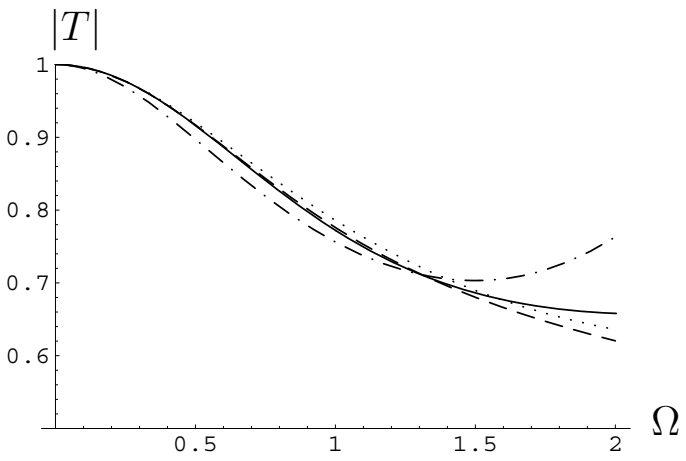

(b) $\phi=45^{\circ}, h=0.4 \mathrm{~m}$.

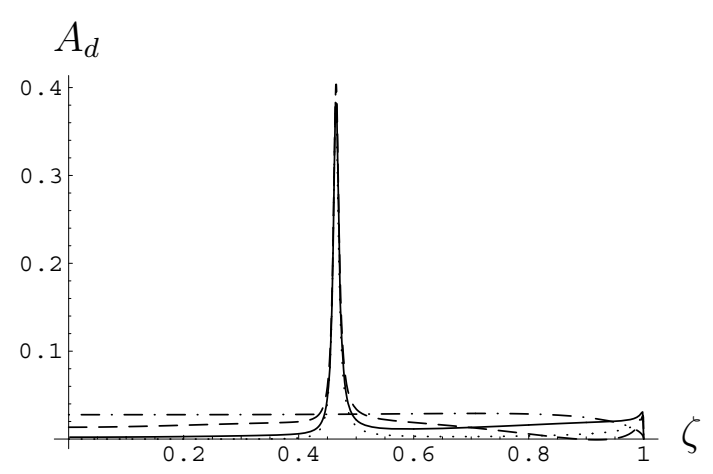

(d) $\Omega=0.8, h=0.04 \mathrm{~m}$.

FIG. 3. Closed pores for QF-20 and water. Exact, $---h^{3}, \cdots h,-\cdot-\mathrm{T}-\mathrm{B}$. 


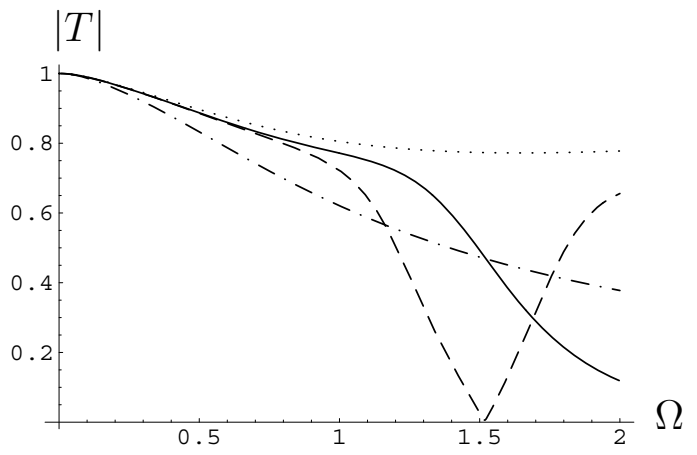

(a) $\phi=15^{\circ}, h=0.04 \mathrm{~m}$.

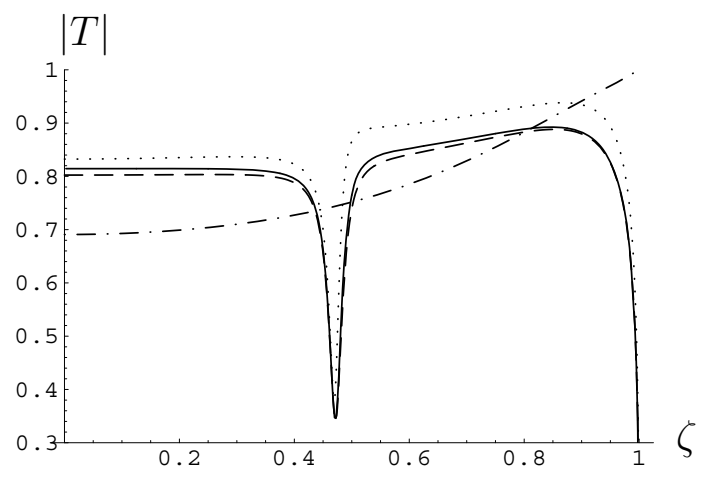

(c) $\Omega=0.8, h=0.04 \mathrm{~m}$.

FIG. 4. Open pores for QF-20 and water.

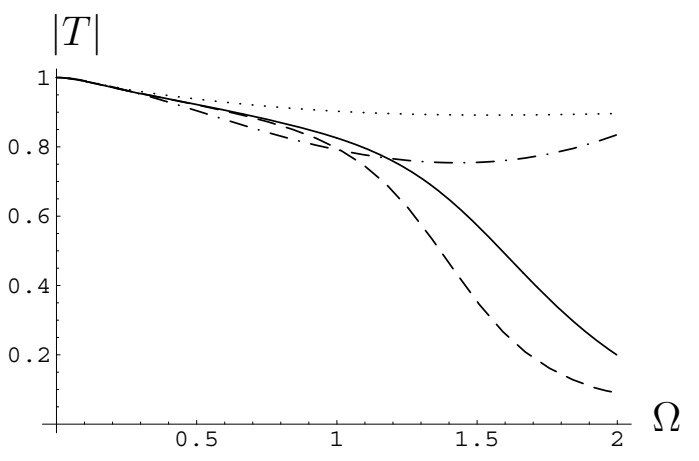

(b) $\phi=45^{\circ}, h=0.04 \mathrm{~m}$.

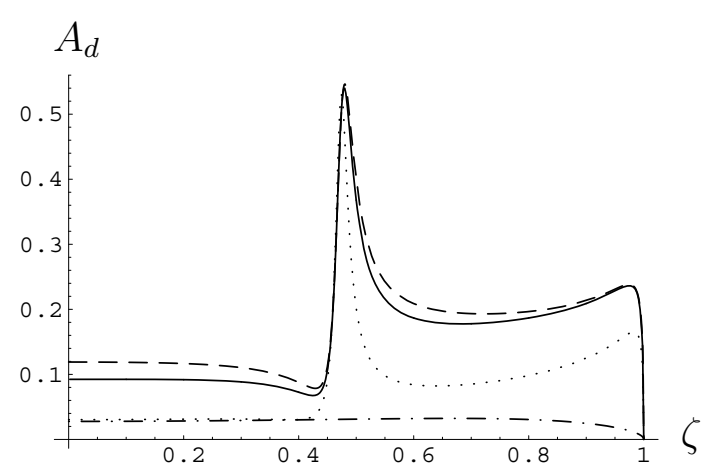

(d) $\Omega=0.8, h=0.04 \mathrm{~m}$.

Exact, $---h^{3}, \cdots h,-\cdot-\mathrm{T}-\mathrm{B}$. 


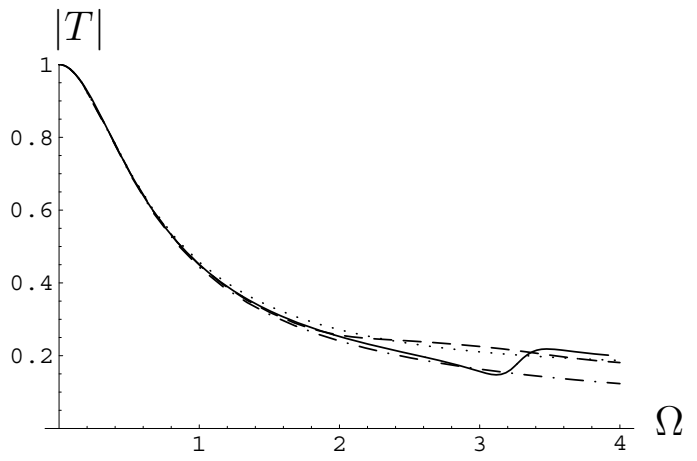

(a) $\phi=45^{\circ}, h=0.04 \mathrm{~m}$.

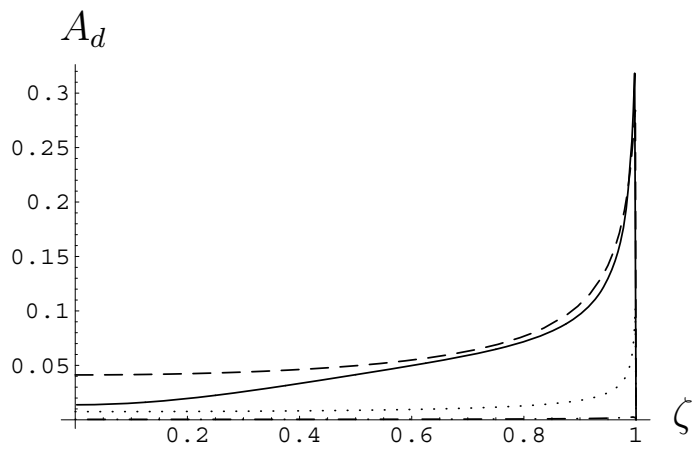

(c) $\Omega=3, h=0.04 \mathrm{~m}$.

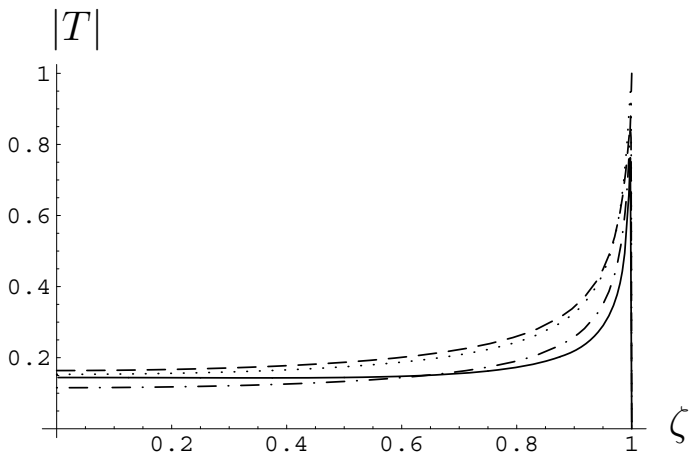

(b) $\Omega=3, h=0.04 \mathrm{~m}$.

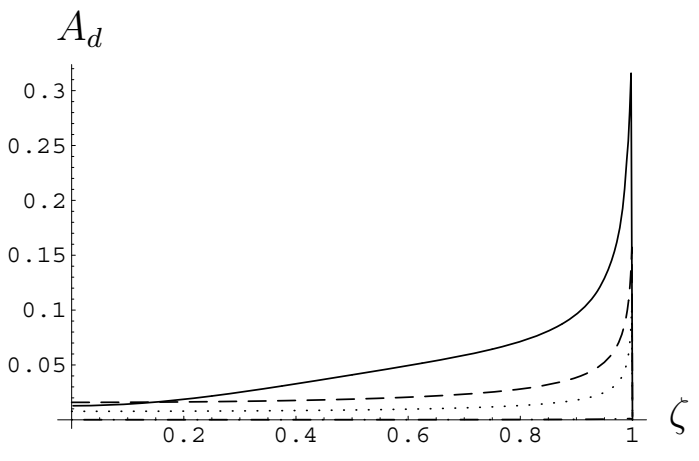

(d) $\Omega=3, h=0.4 \mathrm{~m}$.

FIG. 5. Closed pores for foam and air. Exact, $---h^{3}, \cdots h,-\cdot-$ T-B. 


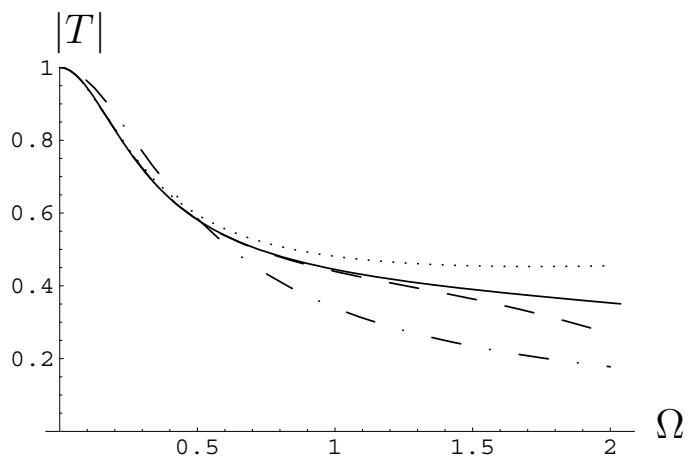

(a) $\phi=45^{\circ}, h=0.04 \mathrm{~m}$.

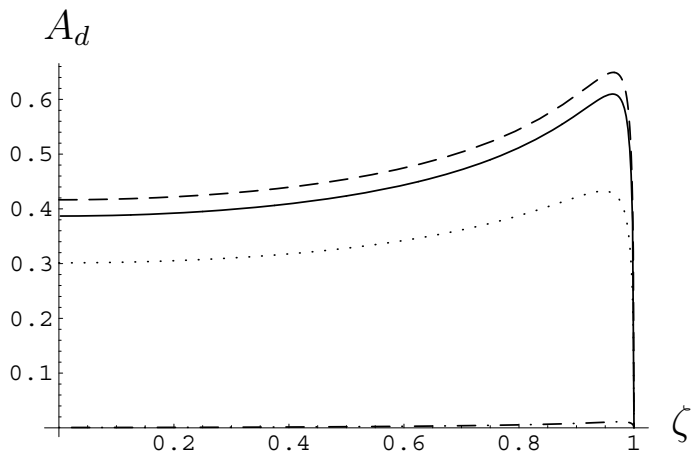

(c) $\Omega=1.5, h=0.04 \mathrm{~m}$.

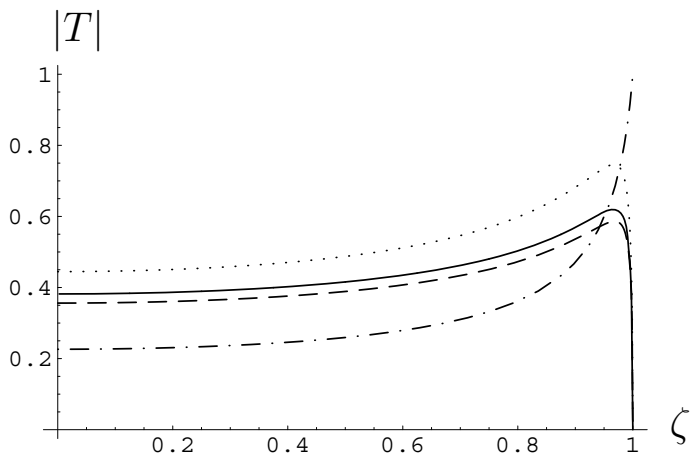

(b) $\Omega=1.5, h=0.04 \mathrm{~m}$.

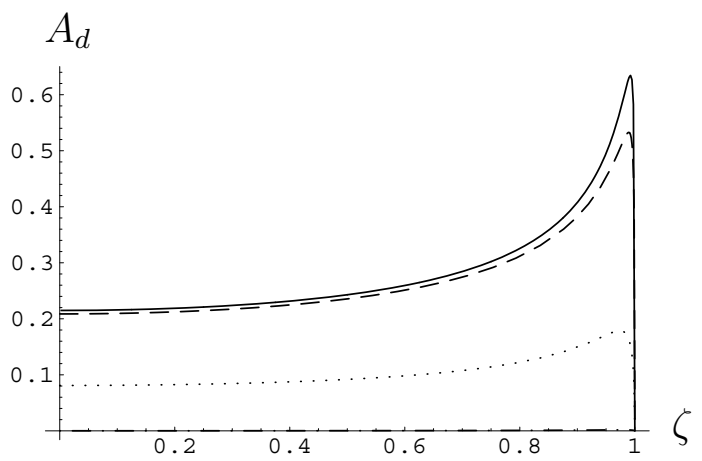

(d) $\Omega=1.5, h=0.4 \mathrm{~m}$.

FIG. 6. Open pores for foam and air. Exact, $---h^{3}, \cdots h,-\cdot-$ T-B. 